\title{
Recombinant Tissue Plasminogen Activator Use in an Acute Stroke Patient With Tuberous Sclerosis
}

\author{
Reza Bavarsad Sahripour ${ }^{\mathrm{a}}$, Ana Hossein Zadeh Malekia, \\ Balaji Krishnaiah $^{\mathrm{a}, \mathrm{b}}$, Andrei Alexandrov ${ }^{\mathrm{a}}$
}

\begin{abstract}
Tuberous sclerosis (TS) is an autosomal dominant disease known for over a century and recognized by characteristic hamartomatous lesions involving any organ. In this report, we are presenting a patient with TS who presented with acute embolic stroke and received tissue plasminogen activator (tPA) and had a mechanical thrombectomy (MT). She had a dramatic response without any hemorrhagic complications. She is a 68-year-old Caucasian woman with the past medical history of TS since childhood who presented to the hospital with symptoms of left middle cerebral artery (MCA) syndrome (right-sided weakness, sensory loss, right facial droop, aphasia, hemianopia, and dysarthria). National Institute of Health Stroke Scale (NIHSS) score was 19 . Head computed tomography (CT) was negative for any acute abnormality except cortical tubers, subependymal ventricular calcification. CT angiography of the head and neck showed proximal occlusion of the left internal carotid artery. After ruling out all exclusion criteria, the patient received tPA and had an MT with complete reperfusion (thrombolysis in cerebral infarction (TICI) 3). Brain magnetic resonance imaging (MRI) showed an acute stroke in the multivessel territories (bilateral parietal cortices, the left lentiform nucleus, medial left temporal lobe, left thalamus, and right occipital lobe) suggestive of an embolic phenomenon and did not show any hemorrhagic complication. TS patients are at increased risk of intracerebral hemorrhage either in the setting of vascular malformation or due to hemorrhage into the subependymal giant cell astrocytoma. The best explanation for the latter could be secondary to elevated venous pressure from increased intracranial pressure. Fortunately, our TS patient who presented with acute ischemic stroke was within the window and received IPA and MT. The patient not only did not have any hemorrhagic side effects afterward but also had a significant improvement in her neurologic symptoms. To our best knowledge, this is the only case report of tPA administration in a TS patient. Moreover, we need more case reports to evaluate the safety of tPA in these patients.
\end{abstract}

Manuscript submitted May 8, 2020, accepted June 10, 2020

Published online July 1, 2020

aDepartment of Neurology, University of Tennessee Health Science Center, Memphis, TN, USA

${ }^{b}$ Corresponding Author: Balaji Krishnaiah, Department of Neurology, University of Tennessee Health Science Center, 847 Monroe Avenue, Suite 218, Memphis, TN 38163, USA. Email: bkrishn4@uthsc.edu

doi: https://doi.org/10.14740/jnr595
Keywords: Tuberous sclerosis; Stroke; Tissue plasminogen; Activator

\section{Introduction}

Tuberous sclerosis (TS) is an autosomal dominant disease known for over a century and recognized by characteristic hamartomatous lesions involving any organ (tuber) [1]. The diagnosis is made by demonstration of its characteristic hamartomas through skin or retina examination, imaging of the viscera, or tissue pathology findings [2]. Given the involvement of the heart and intracranial vessels, these patients are at increased risk of stroke at young age. The stroke could be either hemorrhagic (secondary to intracranial aneurysms) or ischemic (secondary to cardiac rhabdomyoma) [2]. The TS patient is at increased risk of intracranial hemorrhage secondary to underlying cerebrovascular malformation (like ectasia, aneurysm and arteriovenous malformation) or hemorrhage into the subependymal giant cell astrocytoma [3].

In this report, we are presenting a patient with TS who presented with acute embolic stroke who received tissue plasminogen activator (tPA) and had a mechanical thrombectomy (MT). She had a dramatic response without any hemorrhagic complications. To our knowledge, this is the first report of tPA administration in a patient with TS.

\section{Case Report}

A 68-year-old Caucasian woman with the past medical history of TS since childhood, epilepsy, ischemic stroke in 2010, coronary artery disease, systolic heart failure status post automated implantable cardioverter defibrillator (AICD) placement presented to the hospital with symptoms of left middle cerebral artery (MCA) syndrome from $3 \mathrm{~h}$ ago. She had a right-sided weakness, sensory loss, right facial droop, aphasia, hemianopia and dysarthria. National Institute of Health Stroke Scale (NIHSS) score was 19. Head computed tomography (CT) was negative for any acute abnormality except chronic stable diffuse left cerebral hemisphere low-density lesion which reflected cortical tubers, subependymal ventricular calcification and diffuse cerebral volume loss with hydrocephalus ex vacuo. CT 


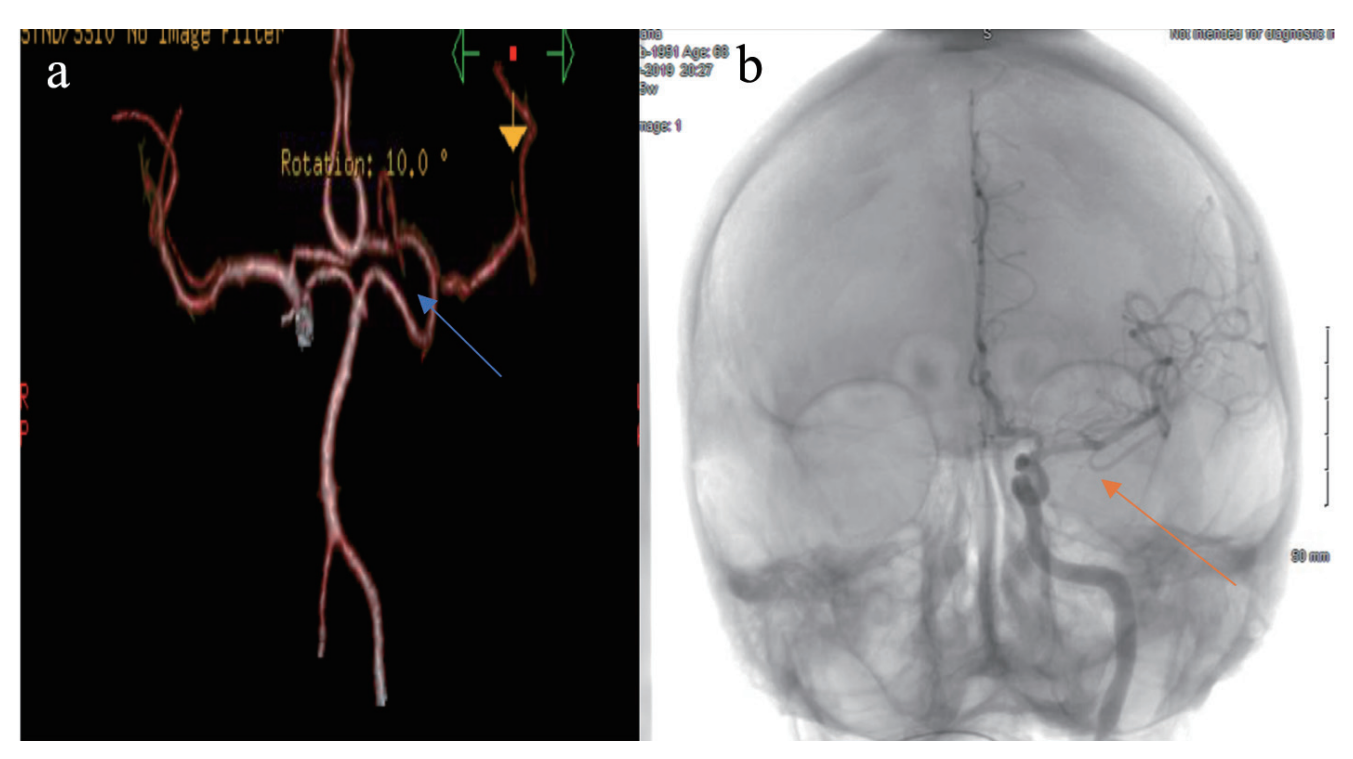

Figure 1. (a) CTA consistent with proximal occlusion, left internal carotid artery and M1 occlusion (blue arrow). (b) DSA showing complete reperfusion $\mathrm{TICl} 3$ (red arrow). CTA: computed tomography angiography; DSA: digital subtraction angiography; TICI: thrombolysis in cerebral infarction.

angiography of head and neck were suggestive of proximal occlusion of the left internal carotid artery (Fig. 1a). The patient received tPA and had an MT (solitaire device) with thrombolysis in cerebral infarction scale (TICI) grade 3 (complete reperfusion) (Fig. 1b). Later her transthoracic echo (TTE) was suggestive of left ventricle ejection fraction of $20-25 \%$ : severe eccentric hypertrophy with global hypokinesis. No left ventricle clots were identified. Severe right and left atrium enlargement were seen.

TTE was suggestive of severe dilatation of right ventricle (RA) with mild systolic dysfunction. Her brain magnetic resonance imaging (MRI) without contrast demonstrated areas of diffusion restriction in multivessel territory involving the bilateral parietal cortices, the left lentiform nucleus, medial left temporal lobe, left thalamus and right occipital lobe suggestive of an embolic phenomenon. Multiple foci of T2 and flair signal hyperintensity seen in the cortices bilaterally are consistent with the tubers (Fig. 2). Patient had good recovery after stroke and her NIHSS score improved to 6 and discharged to rehab unit.

\section{Discussion}

TS is an autosomal dominant neurocutaneous syndrome with variable penetrance. Its frequency is approximately 1 in 6,000 newborns [4]. Although a wide variety of central nervous system (CNS) abnormalities have been recognized to be associated with TS, only a few cases report on ischemic stroke and intracerebral hemorrhage (ICH) are available which are mainly seen in childhood. Seizure and cognitive impairment are the most common CNS presentation of the TS disease. Tubers are the characteristic lesions of TS in the brain, which may rarely differentiate into a malignant subependymal giant cell astrocytoma [3].

TS patients are at increased risk of ICH either in the set- ting of vascular malformation or due to hemorrhage into the subependymal giant cell astrocytoma [3]. The best explanation for the latter could be secondary to elevated venous pressure from increased intracranial pressure $[4,5]$. There are just 16 case reports of intracranial aneurysms in patients with TS and also one case report on cavernous sinus hemorrhage originating from aneurysms of the posterior communicating artery. In most of these cases, the intracerebral aneurysm was an incidental finding and was not associated with any ICH $[3,6]$.

Various heart rhythm and rate abnormalities are seen in individuals with TS, as well as cardiac tumors [2]. The most common primary cardiac tumor in childhood is cardiac rhabdomyoma. Surprisingly studies estimated that up to $70 \%$ to $90 \%$ of children with rhabdomyomas have TS, and at least $50 \%$ of children with TS have rhabdomyomas [2]. In TS patients that present with embolic stroke, the cardiac source should always be suspected, especially in young age, without any known cardiovascular risk factors [7]. Our case had negative echocardiogram for any cardiac tumors and had a heart sinus rhythm with normal rate, but with severely reduced ventricular function.

Fortunately, our TS patient who presented with acute ischemic stroke was within the window and received tPA and MT. Patient not only did not have any hemorrhagic side effect afterwards but also had a significant improvement in her neurologic symptoms.

To our best knowledge, at this time this is the only published case report of TPA administration in a TS patient. Moreover, we need more case reports to evaluate the safety of tPA in these patients.

\section{Acknowledgments}

None to declare. 

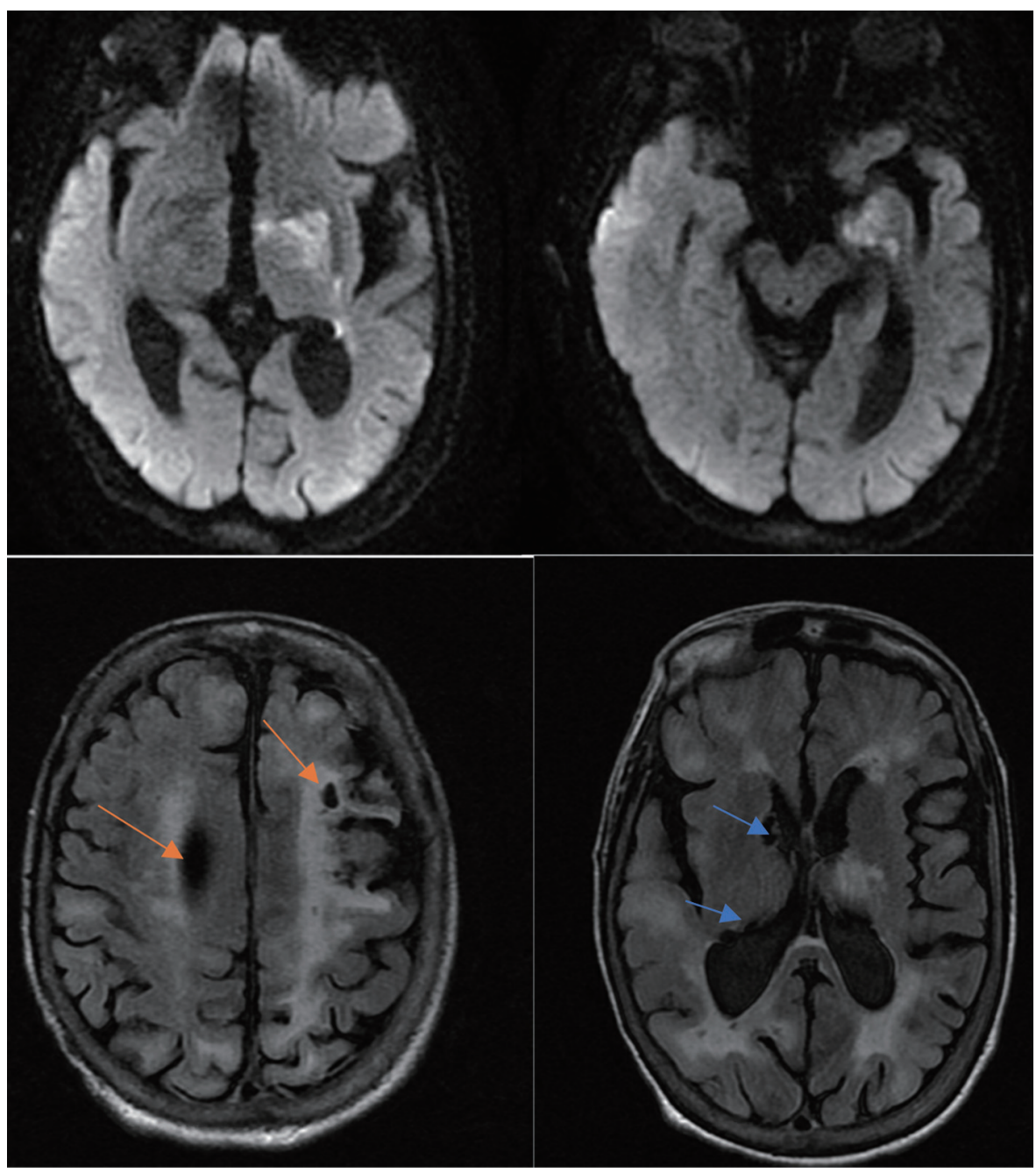

Figure 2. Multifocal restricted diffusion compatible with acute stroke in DWI and multiple cortical tubers (red arrow) and subependymal calcifications (blue arrow) consistent with patient's history of tuberous sclerosis. DWI: diffusion-weighted imaging.

\section{Financial Disclosure}

None to declare.

\section{Conflict of Interest}

None to declare.

\section{Informed Consent}

It was obtained from patient and her family.

\section{Author Contributions}

RBS and AHZM were responsible for designing the case and writing the manuscript; BK and AA contributed to supervising and corresponding.

\section{Data Availability}

The authors declare that data supporting the findings of this study are available within the article.

\section{References}

1. Rodrigues DA, Gomes CM, Costa IM. Tuberous sclerosis complex. An Bras Dermatol. 2012;87(2):184-196.

2. Hinton RB, Prakash A, Romp RL, Krueger DA, Knilans TK, International Tuberous Sclerosis Consensus G. Cardiovascular manifestations of tuberous sclerosis complex and summary of the revised diagnostic criteria and surveillance and management recommendations from the International Tuberous Sclerosis Consensus Group. J Am Heart Assoc. 2014;3(6):e001493. 
3. Yang $\mathrm{L}$, Qin $\mathrm{W}, \mathrm{Hu} \mathrm{W}$. Intracerebral hemorrhage in a patient with tuberous sclerosis complex. Arch Med Sci. 2018;14(4):945-949.

4. Northrup H, Krueger DA, International Tuberous Sclerosis Complex Consensus G. Tuberous sclerosis complex diagnostic criteria update: recommendations of the 2012 Iinternational Tuberous Sclerosis Complex Consensus Conference. Pediatr Neurol. 2013;49(4):243-254.

5. Sabat SB, Cure J, Sullivan J, Gujrathi R. Tuberous sclerosis with multiple intracranial aneurysms: atypical tuber- ous sclerosis diagnosed in adult due to third nerve palsy. Acta Neurol Belg. 2010;110(1):89-92.

6. Hamamoto O, Honorato DC, Brito HL, Souza-Queiroz L. [Intratumor hemorrhage in tuberous sclerosis. A case report]. Arq Neuropsiquiatr. 1994;52(3):435-438.

7. Marx GR, Moran AM. Cardiac tumors. In: Allen HD, Driscoll DJ, Shaddy RE, Feltes TF, eds. Moss and Adams' heart disease in infants, children and adolescents. 7th ed. Chapter 76. New York: Lippincott Williams and Wilkins; 2008: p. 1479-1495. 